SLAC-PUB-8325

January 2000

$\mathrm{UCSD} / \mathrm{PTH}-00-01$

hep-ph/0001172

\title{
Minimal Gaugino Mediation
}

\author{
Martin Schmaltz ${ }^{\mathrm{x}}$ and Witold Skiba ${ }^{\mathrm{y}}$ \\ ${ }^{\mathrm{x}}$ Stanford Linear Accelerator Center, \\ Stanford University, Stanford, CA 94309 \\ ${ }^{\mathrm{y}}$ Department of Physics, \\ University of California at San Diego, \\ La Jolla, CA 92093
}

Submitted to Physics Letters $B$

Stanford Linear Accelerator Center, Stanford University, Stanford, CA 94309

Work supported by Department of Energy contract DE-AC03-76SF00515. 
SLAC-PUB-8325

UCSD/PTH-00-01

hep-ph/0001172

\title{
Minimal Gaugino Mediation
}

\author{
Martin Schmaltz ${ }^{x}$ and Witold Skiba ${ }^{y}$ \\ ${ }^{x} S L A C$, Stanford University, Stanford, CA 94309 \\ schmaltz@slac.stanford.edu \\ ${ }^{y}$ Department of Physics, University of California at San Diego, La Jolla, CA 92093 \\ skiba@einstein.ucsd.edu
}

\begin{abstract}
We propose Minimal Gaugino Mediation as the simplest known solution to the supersymmetric flavor and CP problems. The framework predicts a very minimal structure for the soft parameters at ultra-high energies: gaugino masses are unified and non-vanishing whereas all other soft supersymmetry breaking parameters vanish. We show that this boundary condition naturally arises from a small extra dimension and present a complete model which includes a new extra-dimensional solution to the $\mu$ problem. We briefly discuss the predicted superpartner spectrum as a function of the two parameters of the model. The commonly ignored renormalization group evolution above the GUT scale is crucial to the viability of Minimal Gaugino Mediation but does not introduce new model dependence.
\end{abstract}




\section{INTRODUCTION}

Hidden sectors are an essential ingredient of simple and natural models of supersymmetry (SUSY) breaking. The unifying idea is that SUSY is assumed to be broken in the hidden sector and then communicated to the minimal supersymmetric standard model (MSSM) by messenger interactions which are flavor blind. This structure results in flavor-universal scalar masses and solves the SUSY flavor problem.

In the context of extra dimensions with branes such hidden sectors are very natural. If - for example - the MSSM and the SUSY breaking sector are confined to two different parallel 3-branes embedded in extra dimensions, then the separation in the extra dimensions forbids direct local couplings between the "visible" MSSM fields and the hidden sector. However, fields on separated branes can still communicate by exchanging bulk messenger fields. Couplings which arise from such non-local bulk mode exchange are suppressed. For a messenger of mass $M$ and brane separation $L$ the suppression factor is $e^{-M L}$, which is the Yukawa propagator of the messenger field exchanged between the two branes.

This suggest a very simple scenario for communicating SUSY breaking to the MSSM which guarantees flavor-universal scalar masses. If all light bulk fields have flavor blind couplings then the soft SUSY breaking parameters generated by exchange of these messengers preserve flavor. Heavy bulk modes may violate flavor maximally but the resulting non-universal contributions to the scalar masses are exponentially suppressed $\left[\begin{array}{l}1 \\ 1\end{array}\right]$.

The two obvious candidates for bulk fields which can communicate SUSY breaking to the Standard Model fields in a flavor-blind way are gravity and the Standard Model gauge

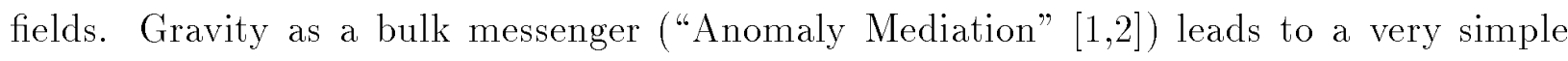
and predictive model which unfortunately predicts negative slepton masses and is therefore ruled out in its simplest and most elegant form $\mathfrak{L}_{-1}^{T_{1}}$ The alternative, Standard Model gauge fields as messengers ("Gaugino Mediation"), has been proposed recently by D.E. Kaplan, Kribs, and Schmaltz [선] to work perfectly. In Gaugino Mediation the MSSM matter fields (quarks, leptons and superpartners) live on a "matter brane", while SUSY breaks on a parallel "SUSY breaking brane", and the MSSM gauge superfields live in the bulk. Because the gaugino fields are bulk fields they couple directly to the SUSY breaking and obtain soft masses. The MSSM scalars are separated from SUSY breaking by the distance $L$ and therefore obtain much smaller masses from non-local loops with high momentum modes of the bulk gauge fields

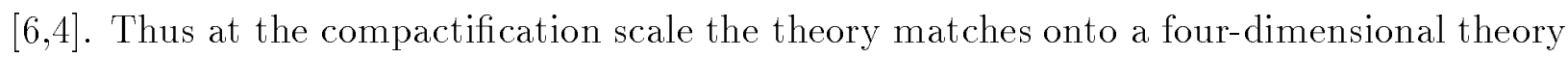
with gaugino masses and negligibly small scalar masses.

Vanishing scalar masses and non-vanishing gaugino masses at a high scale, as in noscale models [i]1] , is very attractive because evolving the theory to low energies via the

\footnotetext{
${ }^{1}$ For models which cure Anomaly Mediation by introducing new fields and interactions see [3.3.3.
} 
renormalization group equation generates flavor-universal and positive soft scalar masses. Consistent electroweak symmetry breaking also requires a $\mu$ term of size comparable to the gaugino masses. Thus a minimal version of Gaugino Mediation has only three high energy parameters

$$
\mu, \quad M_{1 / 2}, \quad M_{c}
$$

Here $M_{1 / 2}$ is the common gaugino mass at the unification scale, and $M_{c}$ is the compactification scale where the higher dimensional theory is matched onto the effective four-dimensional theory. Since we wish to preserve the successful prediction of $\sin ^{2} \theta_{w}$ from gauge coupling unification in the MSSM we limit $M_{c}>M_{G U T}$.

In Section 'Tl' of this paper we show that this scenario, which we call "Minimal Gaugino Mediation" (Mg̃ M), with only the parameters in Eq. (i.jili $)$ works very well phenomenologically. The minimal scenario which we advocate here differs from the more general models

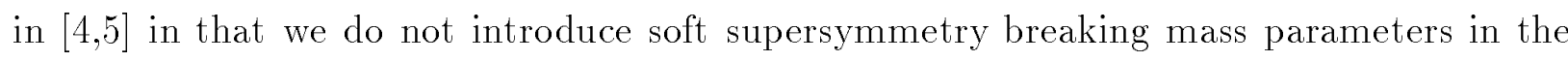
Higgs sector of the theory at $M_{c}$. Radiative electroweak symmetry breaking works automatically in M grM and determines $\mu$ by fitting to the $Z$ mass. Therefore the entire superpartner spectrum of $\mathrm{Mg} \mathrm{M}$ can be computed via the renormalization group equations in terms of only two free parameters: $M_{1 / 2}$ and $M_{c}$. We will see that the running from $M_{c}$ to $M_{G U T}$ in the grand unified theory is important for the masses of the lightest superpartners. We find that the Bino is the LSP and a perfect cold dark matter candidate in a large region of the models" parameter space. "Minimal Gaugino Mediation" also evades all existing collider bounds without fine-tuning.

In Section III we present a complete and economical model which gives rise to the Mg̃ M boundary condition. The model generates the hierarchy between the Planck scale and the SUSY breaking scale with the extra-dimensional dynamical supersymmetry breaking mechanism of Arkani-Hamed, Hall, Smith, and Weiner [s]. To solve the $\mu$ problem without introducing a $B \mu$ problem we propose a new mechanism in which five-dimensional $N=1$ supersymmetry relates $\mu$ and the gaugino mass.

In Section IVI! we briefly explain why Mg̃M has no SUSY CP problem, estimate the neutralino relic density, and conclude.

\section{SPARTICLE SPECTRUM IN M $\tilde{g} M$}

In this section we determine the predictions of $\mathrm{Mg} M$ for the spectrum of MSSM particles. The input parameters of the model are listed in Eq. (1.1 $\left.\overline{1}_{1}\right)$. We use the renormalization group equations (RGEs) of the $\overline{\mathrm{DR}}$ scheme to calculate the soft breaking parameters at the electroweak scale. We first outline our procedure for the running and discuss general features of the evolution. Then we present the spectrum of superparticles and describe how the experimental limits translate into constraints on the parameter space of the model. 
At the compactification scale $M_{G U T} \lesssim M_{c} \lesssim M_{\text {Planck }} / 10$ the mass parameters of $\mathrm{Mg} \mathrm{M}$ are

$$
M_{1 / 2} \sim \mu \neq 0, \quad m^{2}=A=B=0 .
$$

We limit the range of compactification scales from below by the GUT scale in order to preserve the successful prediction of $\sin ^{2} \theta_{w}$ from four dimensional unification in the MSSM. Note that this requirement would still allow compactification scales slightly below $M_{G U T}$; however as we will discover below, $M_{c}$ needs to be slightly larger than $M_{G U T}$ to avoid a charged LSP. The upper limit on $M_{c}$ is more model dependent. It arises from demanding that flavor violating soft masses are sufficiently small. Such masses are generated from exchange of massive bulk fields with flavor-violating couplings, which are expected to be present in any fundamental theory which explains the Yukawa couplings of the Standard Model. Assuming that the lightest such states have masses of order $M_{\text {Planck }}$ the suppression factor is of the order of $\exp \left(-M_{\text {Planck }} / M_{c}\right)$. Requiring that this exponential suppresses off-diagonal squark masses sufficiently gives $M_{c} \lesssim M_{\text {Planck }} / 10$.

To connect the boundary condition of Eq. $\left(2 . \overline{1}_{1}\right)$ to experiments at the weak scale we first run from $M_{c}$ to $M_{G U T}$ in the unified theory and then run from $M_{G U T}$ to the weak scale with the RGEs of the MSSM. Gaugino domination, or the no-scale, boundary conditions have been studied extensively in the literature, however only including renormalization below the GUT scale $\left[\begin{array}{ll}\overline{0} \\ 0\end{array}\right]$. Since the renormalization effects above the GUT scale are not discussed very frequently in the literature we describe them in some detail first.

Naively, one might be tempted to argue against calculating renormalization effects above the GUT scale because: $i$. the running above the GUT scale gives only very small masses because $\log \left(\frac{M_{c}}{M_{G U T}}\right) \ll \log \left(\frac{M_{G U T}}{M_{\text {weak }}}\right)$ and $i i$. the running of soft masses above the GUT scale is model dependent because the theory above the GUT scale contains new unknown fields and couplings which enter the RGEs and give rise to unknown threshold effects. Both of these arguments are invalid as is easy to see: Argument $i$. neglects group theory factors. For example, the mass which is generated for the right-handed sleptons from running below the GUT scale is very small because they only couple to hypercharge. Above the GUT scale, sleptons are unified into larger GUT representations and the associated larger multiplicity factors more then compensate for the smaller log. The second argument would apply in general theories with soft masses, but it does not apply to Mg̃M where (at one loop) all generated soft masses are determined by gauge charges only. To understand this consider a generic one-loop RG equation for scalar soft terms

$$
\frac{d}{d t}(\text { soft }) \propto g^{2} M_{1 / 2}+(\text { soft }) f\left(g^{2}, \text { SUSY couplings }\right) .
$$

Here the first term is determined entirely by the known gauge charges, whereas the second term depends on unknown new fields and couplings. However, in M g̃ M all soft terms for the scalars are zero at $M_{c}$. Therefore, the soft masses appearing in the second term are small 
(loop-suppressed compared to $M_{1 / 2}$ ), and it is a good approximation to drop the second term. The only remaining model dependence is in the gauge interactions above the GUT scale. The predictions depend on the choice of unified gauge group, and we present predictions for both $S U(5)$ and $S O(10)$. Furthermore, there is also a weak dependence on the running of the unified gauge coupling above the GUT scale. We perform our renormalization group analysis assuming a minimal set of GUT representations $(3 \times(10+\overline{5})+5+\overline{5}+24$ for the case of $S U(5)$ and $3 \times 16+10+45+16+\overline{1} 6$ for $S O(10))$. However, even adding as much as three additional adjoints to either theory would change the final scalar masses by at most a few percent $L_{L 1}^{L_{2}}$ Finally, note that GUT threshold corrections to the supersymmetry breaking scalar masses vanish in $\overline{\mathrm{DR}}$ so that using the RGEs gives the complete answer.

Even though we perform our renormalization group analysis numerically one can also obtain extremely simple approximate formulae for the soft parameters at the GUT scale as follows. At one loop the ratio $\frac{M_{1 / 2}}{g^{2}}$ is RGE invariant. Thus, the running of $M_{1 / 2}$ is trivial as it traces the running of the gauge coupling, and we present our results using $\alpha$ and $M_{1 / 2}$ evaluated at $M_{G U T}$ rather than at $M_{c}$. Assuming that the running of the couplings above the GUT scale is not too fast all other soft terms at the GUT scale are then given by [i]

$$
\begin{aligned}
A_{\text {top }} & =-\frac{2 \alpha}{\pi} M_{1 / 2} t_{c}\left[\frac{24}{5}, \frac{63}{8}\right], \\
B & =-\frac{2 \alpha}{\pi} M_{1 / 2} t_{c}\left[\frac{12}{5}, \frac{9}{2}\right], \\
m_{\overline{5}}^{2} & =\frac{2 \alpha}{\pi} M_{1 / 2}^{2} t_{c}\left[\frac{12}{5}, \frac{9}{2}\right], \\
m_{\mathbf{1 0}}^{2} & =\frac{2 \alpha}{\pi} M_{1 / 2}^{2} t_{c}\left[\frac{18}{5}, \frac{45}{8}\right],
\end{aligned}
$$

where $t_{c}=\log \left(\frac{M_{c}}{M_{G U T}}\right)$ ranges between 0 and 4 . Note that we defined the trilinear soft scalar coupling as $A_{\text {top }} \cdot Y_{\text {top }}$. All parameters in the equations above are evaluated at the GUT scale. The gauge coupling at the unification scale is determined from the low-energy values of the couplings and it corresponds to $\alpha_{G U T}=1 / 24.3$. The first set of numbers in parenthesis applies to $S U(5)$, the second one to $S O(10)$. With an abuse of notation for the case of $S O(10)$ we defined $m_{\overline{5}}^{2}$ to denote the soft mass for the Higgses of the MSSM, while $m_{\mathbf{1 0}}^{2}$ denotes the common soft mass of the matter fields.

As we will see shortly, Mg̃ M with $S U(5)$ unification predicts moderate values of $\tan \beta$ between 12 and 25 so that we can neglect the effect of the bottom Yukawa coupling on the running of soft parameters. For $S O(10)$ larger values of $\tan \beta$ arise for large compactification scales. Since we have only kept the top Yukawa in our RGEs we limit $t_{c} \leq 2$ for $S O(10)$. Then the only significant $A$ term generated is $A_{\text {top }}$.

Below the GUT scale we integrate the one-loop RGEs [i] numerically. One loop-running

\footnotetext{
${ }^{2} \mathrm{~A}$ more detailed discussion of RGEs above the GUT scale can be found in
} 
has adequate precision if one uses the one-loop improved Higgs potential [i 5. The dominant correction to the lightest Higgs mass comes from top quark loops below the stop mass threshold. It can be accounted for by adding the term

$$
\frac{3 Y_{\text {top }}^{4}}{16 \pi^{2}} \log \frac{m_{\tilde{t}_{L}} m_{\tilde{t}_{R}}}{m_{t}^{2}}\left(H_{u}^{\dagger} H_{u}\right)^{2} .
$$

In addition, we incorporate the contributions to squark and slepton masses arising from D-terms as described in Ref. [i] $\mathbf{1}^{i}$.

After evolving all soft masses to the weak scale we impose the constraints which follow from radiative electroweak symmetry breaking. This determines the weak scale values of both $\mu$ and $\tan \beta$, and we are left with only two free parameters: $M_{1 / 2}\left(M_{G U T}\right)$ and the compactification scale $M_{c}$. The $\mu$ parameter is multiplicatively renormalized, and it does not enter any RGE at one loop. Therefore, we will quote its value at the weak scale.

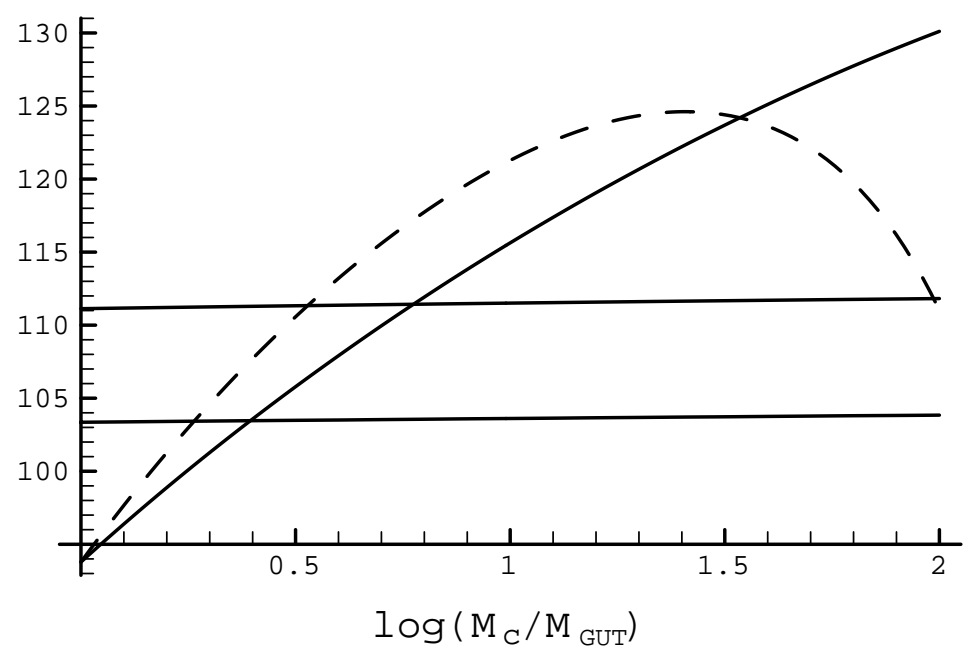

FIG. 1. The dependence of the masses of the stau, the lightest neutralino and the lightest Higgs on the compactification scale for fixed $M_{1 / 2}=250 \mathrm{GeV}$. The neutralino mass $(104 \mathrm{GeV})$ and the Higgs mass $(111 \mathrm{GeV})$ are almost independent of the compactification scale and the GUT gauge group. The rising solid line and the dashed line indicate the mass of the stau in $S U(5)$ and $S O(10)$, respectively.

Figure $i_{-1}^{1}$ illustrates the significance of the RG evolution above the GUT scale. Without running above the GUT scale $\left(M_{c}=M_{G U T}\right)$ the stau is the LSP; however for any compactification scale larger than only $1.5 M_{G U T}$ the stau is heavier than the lightest neutralino. Note that the dependence of the stau mass on $t_{c}=\log \left(\frac{M_{c}}{M_{G U T}}\right)$ is stronger in $S O(10)$ than in $S U(5)$. This follows from the larger group theoretical factors in $S O(10)$ which cause soft masses above the GUT scale to be generated more efficiently.

The allowed parameter space for $S U(5)$ and $S O(10) \mathrm{Mg} \mathrm{M}$ models is presented in Figure We find a lower bound on $t_{c}$ from requiring that the LSP be neutral. An upper bound on $t_{c}$ is not shown on the figure, but as discussed above, flavor violating effects due to massive bulk fields limit $t_{c} \lesssim 4$. Since $M_{1 / 2}$ is the only source for superpartner masses, the experimental 

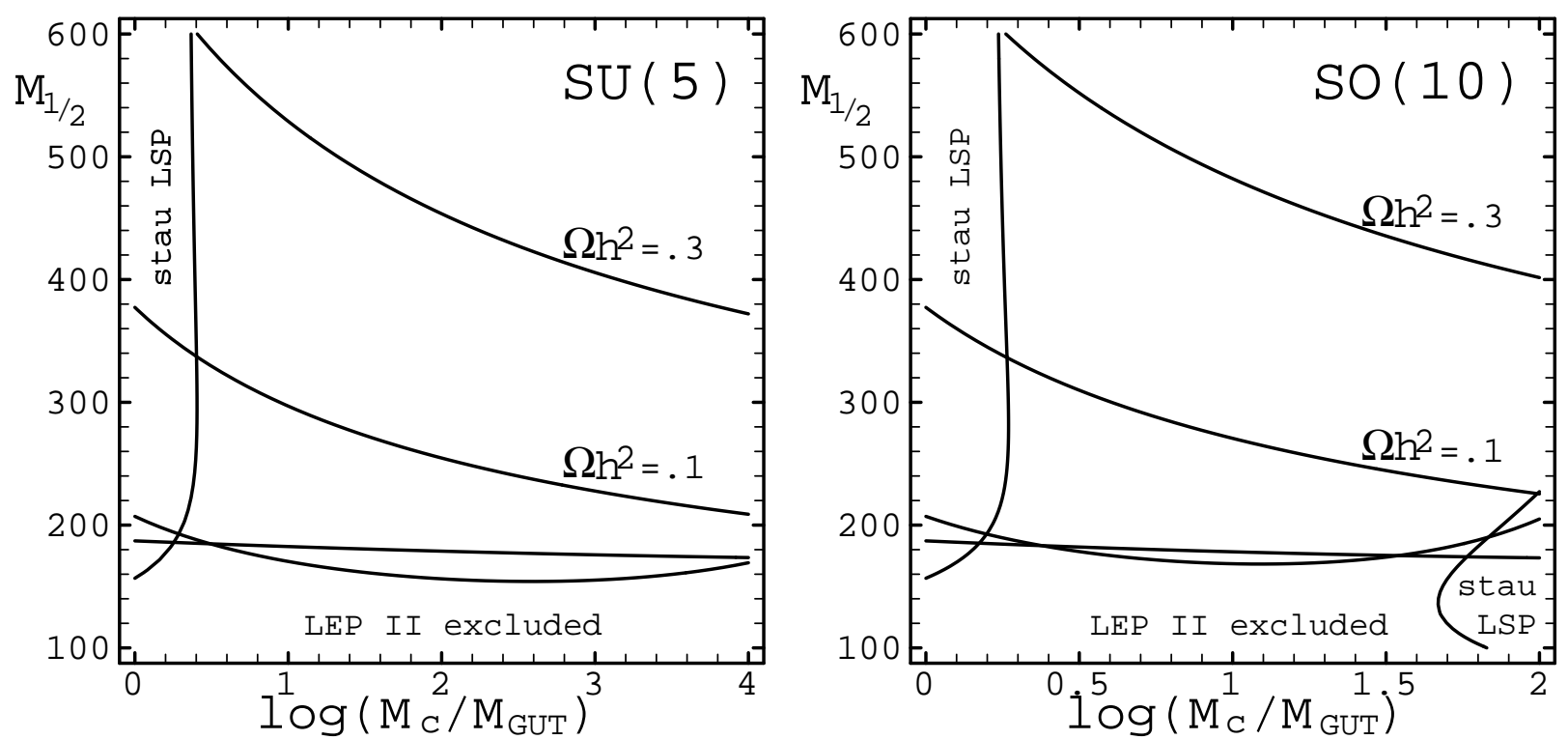

FIG. 2. The allowed region of the parameter space for Mg̃M with $S U(5)$ and $S O(10)$ unified group. The curves at the bottom of the figure correspond to LEP II limits on the masses of the Higgs (straight line) and stau (concave). Demanding that relic LSPs contribute the cosmologically preferred amount of cold dark matter and do not over-close the universe singles out the region of parameter space between the lines labeled $\Omega h^{2}=0.1$ and 0.3 .

lower limits on superpartner masses and the Higgs mass translate into lower limits on $M_{1 / 2}$.

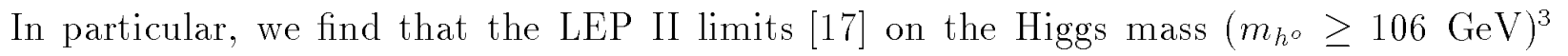
and the right handed slepton masses $\left(m_{\tilde{\tau}} \geq 75 \mathrm{GeV}\right.$ and $\left.m_{\tilde{e}} \geq 95 \mathrm{GeV}\right)$ imply $M_{1 / 2} \gtrsim 180$ $\mathrm{GeV}$. Furthermore, we find that the $\mu$ parameter in our model is given by $\mu=3 / 2 M_{1 / 2}$ to an accuracy of better than $2 \%$ for all values of $t_{c}$. This implies a lower bound $\mu \gtrsim 270$ $\mathrm{GeV}$ with an associated mild tuning of the $Z$ mass. The figure also shows contours of the relic abundance of the lightest neutralino corresponding to $\Omega_{\chi} h^{2}=0.1,0.3$. The LSP relic abundance calculation is particularly simple in our model, we discuss it briefly in Section IV:

Figure ${ }_{3}^{-1}$ shows the Mğ spectrum as a function of the gaugino mass for the example case of an $S U(5)$ GUT with $\log \left(\frac{M_{c}}{M_{G U T}}\right)=2$. The qualitative features of the spectrum are generic and do not depend on the choice of grand unified group or compactification scale. The masses of all superpartners and Higgs fields, except for the lightest Higgs, rise linearly with $M_{1 / 2}$. As in minimal supergravity the LSP is a Bino-like neutralino. The right-handed stau is the next-to-LSP. As usual, colored superpartners are heaviest, followed by charginos, neutralinos and Higgses with masses of order $\mu$. The mass of the lightest Higgs particle increases only logarithmically with $M_{1 / 2}$ through the one-loop improvement of the Higgs

\footnotetext{
${ }^{3}$ The Standard Model Higgs bound rather than the much weaker SUSY Higgs bound applies in the entire allowed parameter space because $\mu$ is sufficiently large so that the heavier Higgs fields decouple and the production cross section becomes Standard-Model-like.
} 

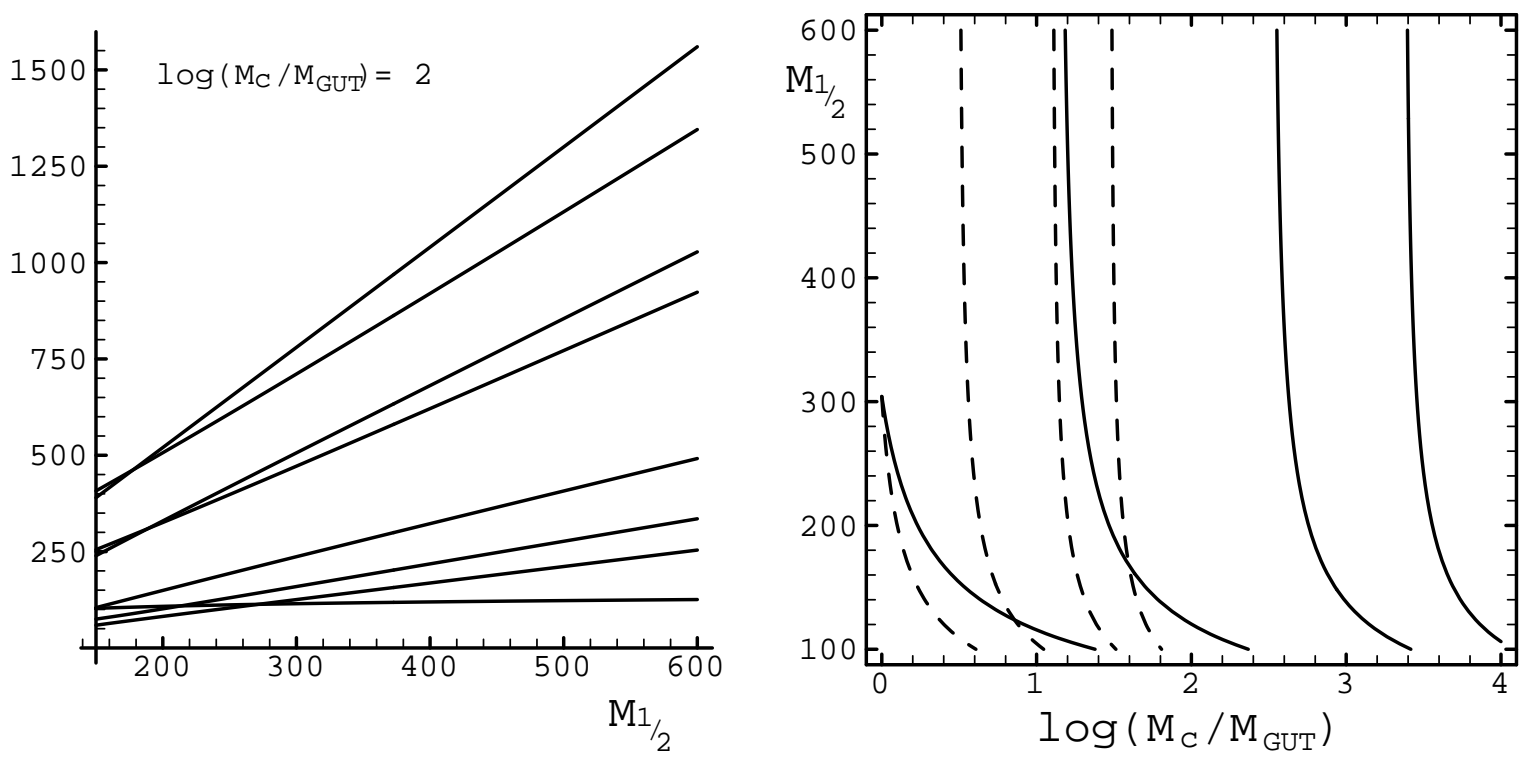

FIG. 3. Left graph: particle masses as functions of $M_{1 / 2}$ for fixed compactification scale. The lines correspond to (from lightest to heaviest at $M_{1 / 2}=600 \mathrm{GeV}$ ) the lightest Higgs, lightest neutralino, right-handed stau, second lightest neutralino, heavy chargino, pseudoscalar Higgs, left-handed stop, and gluino. Right graph: contours of constant $\tan \beta$. The contours correspond to $\tan \beta$ of $12,15,20$, and 25 from left to right. The solid lines are for $S U(5)$, the dashed ones for $S O(10)$.

potential as described in Eq. (15.7.

Figure $\overline{1}$ also shows contours of constant $\tan \beta$ in the $M_{1 / 2}-t_{c}$ plane. Note that in the allowed region $\tan \beta$ is almost independent of $M_{1 / 2}$, but it increases with $t_{c}$.

Since M ğM has only 2 free parameters, measuring the masses of only two particles is in principle sufficient to determine the input parameters and predict the entire superpartner spectrum. In practice, presumably the Higgs will be the first new particle to be discovered. This is because the MSSM Higgs mass bound of $130 \mathrm{GeV}$ applies also to the Mg̃ M Higgs which could therefore be discovered (or ruled out) at Run II of the Tevatron [i] $\bar{s}_{-1}^{1}$, and might even be seen at LEP 205. The mass of the Higgs would give an estimate of $M_{1 / 2}$. Should $M_{1 / 2}$ be close to $200 \mathrm{GeV}$, there is a chance that LEP or the Tevatron will discover the first superpartners. For low enough compactification scales LEP would find the right-handed stau and/or selectron. Independent of the compactification scale the Tevatron could then observe charginos in the tri-lepton channel [i] $\left.{ }_{1}^{1}\right]$. For larger $M_{1 / 2}$ we would have to wait for the LHC.

It is exciting that observation of the first superpartner immediately also leads to a first test of the model. This is because the discovery would allow a mass measurement of both the discovered superpartner as well as the LSP mass from the distribution of the missing energy. One could then use the measured masses of the Higgs and Bino to obtain two independent determinations of $M_{1 / 2}$ and therefore test the model. Once we know a mass of any of the sleptons we can extract the remaining free parameter $-t_{c}$. Note that discovery of just a few of the lightest superpartners would already allow a determination of the GUT gauge group! 


\section{M $\tilde{g} M, A N$ EXPLICIT MODEL}

In this section we describe a simple model which breaks supersymmetry and yields only gaugino masses at the compactification scale. Our model is complete: it generates exponentially small supersymmetry breaking which is mediated to the gauginos via a higher dimensional operator, and $\mu$ is naturally of the same order as the gaugino masses. The model combines the idea of "gaugino mediation" with the supersymmetry breaking mechanism proposed in [يil].

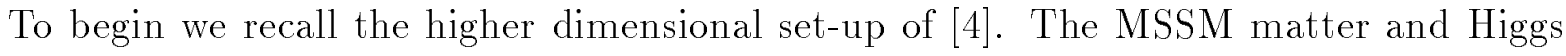
fields live on a $3+1$ dimensional brane embedded in one extra dimension. Supersymmetry is broken dynamically on a parallel brane which is a distance $L$ apart from the matter brane (Fig. 位). The MSSM gauge fields and gauginos live in the bulk of the extra dimension. We take this extra dimension to be circular, with radius $R$. In order to preserve the quantitative prediction of $\sin ^{2} \theta_{w}$ from gauge coupling unification in the four dimensional MSSM, we demand that the compactification scale be higher than the GUT scale, $R^{-1} \equiv M_{c} \geq M_{G U T} \cdot \stackrel{\text { In }}{ }^{\prime}$

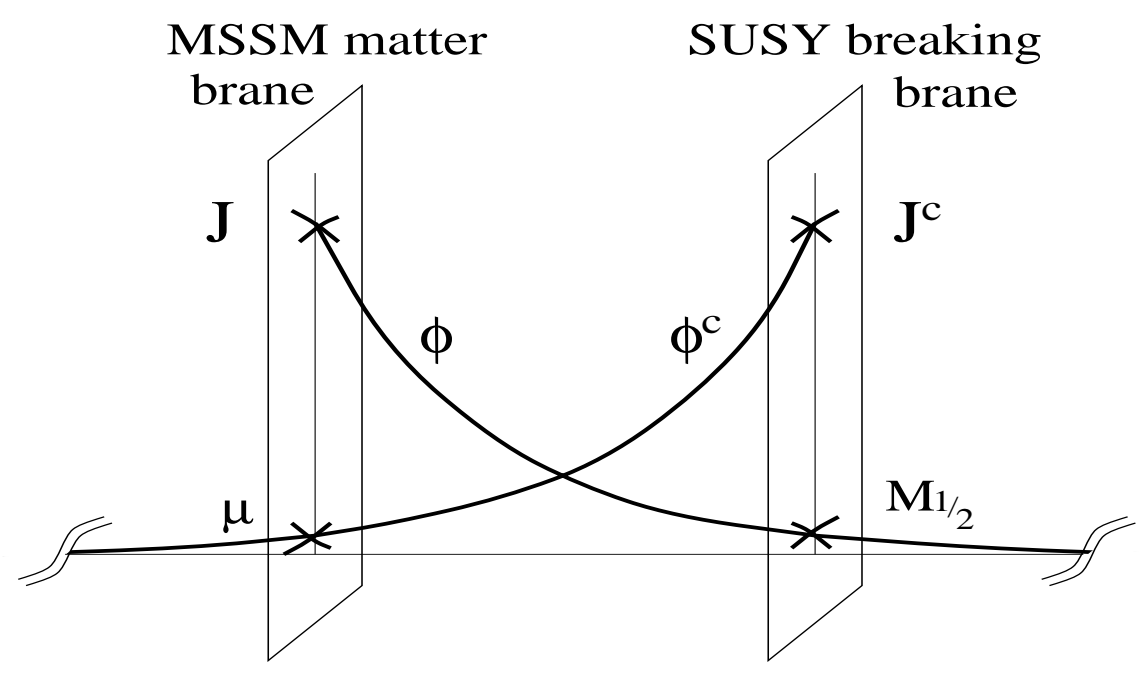

FIG. 4. A brane configuration which leads to the Minimal Gaugino Mediation boundary condition. Pictured is the extra dimension from left to right with periodic boundary conditions. We also show the exponentially decaying vacuum expectation values of $\phi$ and $\phi^{c}$ which are responsible for generating hierarchically small supersymmetry breaking and the $\mu$ term.

In our model, supersymmetry breaking manifests itself in a vacuum expectation value for the F-component, $X_{F}$, of a chiral superfield $X$ on the SUSY breaking brane. The MSSM gaugino fields can couple to $X$ directly, giving a gaugino mass

\footnotetext{
${ }^{4}$ A similar constraint on the compactification scale also follows from demanding that the extradimensional theory remains perturbative up to the five dimensional Planck scale $M, g_{G U T}^{2}=\frac{g_{5}^{2}}{2 \pi R}<$ $\frac{24 \pi^{5 / 2}}{2 \pi R M}$. Using $2 \pi R M^{3}=M_{\text {Planck }}^{2}$ this becomes $R M_{\text {Planck }}<750$.
} 


$$
\int d x_{5} \delta\left(x_{5}-L\right) \int d^{2} \theta \frac{X W W}{M^{2}} \rightarrow \frac{X_{F}}{V M^{2}} \lambda \lambda
$$

Here, the factor of the extra-dimensional volume ( $V=2 \pi R$ for a circle) arises from the wave function normalizations of the four-dimensional gaugino fields $\lambda$. All other soft supersymmetry breaking parameters in the MSSM, such as soft scalar masses $X^{\dagger} X Q^{\dagger} Q$, are suppressed at short distances by extra-dimensional locality [셧. The low-energy values of these parameters are generated from the renormalization group equations as discussed in the previous section.

It is useful to discuss the exact form of the short-distance suppressions in more detail. In general, there are two possible sources for such terms: direct contact terms suppressed by the cut-off $M$ or non-local terms from loops of the light bulk gauge fields. The contact terms are not present in the effective theory below $M$ to all orders in the local expansion in inverse powers of $M$ because they connect fields at different positions. However, this does not preclude the appearance of terms with coefficients $e^{-L M}$ which do not have an expansion in local operators. These operators are expected to be flavor violating and are therefore strongly constrained experimentally [i20i]. The most stringent constraint comes from $\mathrm{CP}$ violation in the $\mathrm{K}$ system and gives roughly $e^{-L M} \lesssim 10^{-4}$ or $L M \gtrsim 8$. Therefore, the allowed range for the compactification scale is $M_{G U T} \lesssim M_{c} \lesssim M_{\text {Planck }} / 10$.

The other source of short-distance scalar masses - loops of bulk gauge fields - leads to finite contributions to the masses which are suppressed by additional powers of the separation $L$ relative to the gaugino mass ( $\left(3 . \overline{1} \mathbf{1}_{1}\right)$. However, they are flavor universal because they arise from gauge interactions. As discussed in detail in [A] these contributions are negligible compared to the much larger contributions from the renormalization group evolution.

In the following Subsections we turn to discussing the mechanism of supersymmetry breaking and the origin of the $\mu$ term in the model. Our mechanism for breaking supersymmetry and stabilizing the radius of the extra dimension is taken directly from the elegant paper of Arkani-Hamed et. al. [is]. In the following, we summarize their discussion and apply it to our model. Our solution to the $\mu$ problem is new.

\section{A. Supersymmetry breaking}

Following [8], we keep track of four-dimensional $N=1$ supersymmetry by employing four-dimensional $N=1$ superspace notation and treating the $x_{5}$ coordinate as a label. The action for a massive five-dimensional hypermultiplet $\left(\Phi, \Phi^{c}\right)$ then reads

$$
\int d^{4} x d x_{5}\left(\int d^{4} \theta\left(\Phi^{\dagger} \Phi+\Phi^{c \dagger} \Phi^{c}\right)+\int d^{2} \theta \Phi^{c}\left(m+\partial_{5}\right) \Phi\right) .
$$

The advantage of this formalism is that it is straightforward to write down $N=1$ supersymmetric couplings of $\Phi$ to boundary fields. The supersymmetry breaking model of [sis consists of the bulk field $\Phi$ with superpotential couplings to a source $J$ and a field $X$ which 
are localized on different branes. $J$ is localized on the matter brane at $x_{5}=0$, while $X$ on the SUSY-breaking brane at $x_{5}=L$

$$
\int d x_{5}\left(-\delta\left(x_{5}\right) \sqrt{M} J \Phi^{c}+\delta\left(x_{5}-L\right) \sqrt{M} X \Phi\right) .
$$

Here we have suppressed coupling constants but inserted factors of the fundamental mass scale $M$ to keep track of mass dimensions. The vacuum equations for the scalar field are then

$$
\begin{aligned}
& \Phi_{F}=\delta\left(x_{5}-L\right) \sqrt{M} X+\left(m-\partial_{5}\right) \phi^{c}=0, \\
& \Phi_{F}^{c}=-\delta\left(x_{5}\right) \sqrt{M} J+\left(m+\partial_{5}\right) \phi=0 .
\end{aligned}
$$

On a circle $x_{5} \in[0,2 \pi R)$ the equation for $\Phi$ has the unique solution

$$
\phi=\sqrt{M} J \frac{e^{-m x_{5}}}{1-e^{-m 2 \pi R}} .
$$

Thus the source $J$ "shines" a vacuum expectation value for the bulk scalar $\phi$ which decays exponentially with increasing $x_{5}$ (see Fig. 高). Supersymmetry is broken because $X$ obtains a non-vanishing $F$-component

$$
X_{F}=\sqrt{M} \phi(L)=M J \frac{e^{-m L}}{1-e^{-m 2 \pi R}} \sim M J e^{-m L} .
$$

Assuming a source $J \sim M$ and a mass $m \sim M$ one finds $X_{F} \sim M^{2} e^{-M L}$.

Note that this model is a higher dimensional generalization of a simple O'Raifeartaigh model. The source $J$ forces a non-zero expectation value for the field $\phi$, that is in conflict with the $X$ equation of motion which requires $\phi=0$. The role of the extra dimension is to modulate the resulting supersymmetry breaking by the factor $e^{-M L}$. Coupling the field $X$ to the gauge fields as in Eq. ( $\left(3 . \overline{1}_{1}\right)$ then results in non-vanishing gaugino masses

$$
M_{1 / 2}=\frac{X_{F}}{2 \pi R M^{2}} \sim \frac{J}{2 \pi R M} e^{-M L} .
$$

As in ordinary O'Raifeartaigh models, the scalar expectation value of $X$ is undetermined classically. A non-vanishing expectation value can be seen to act as a source for $\Phi^{c}$ from Eq. $\left(\overline{3}-4^{\prime}\right)$. In order to simplify the analysis we assume that the $X$-expectation value is zero. This may either be enforced by additional tree-level superpotential terms on the supersymmetry breaking brane such as $\delta\left(x_{5}-L\right)\left[X Y+Y^{2} Z\right]$, or it could be a result of quantum corrections lifting the flat direction.

\section{B. The $\mu$ term}

To generate a $\mu$ term of the correct size we utilize the $\phi^{c}$ component of the superfield $\left(\Phi, \Phi^{c}\right)$. To break supersymmetry we used an expectation value for $\phi$ which was "shining" 
clockwise from the source $J$ on the matter brane towards the supersymmetry breaking brane. For generating $\mu$ we "shine" an expectation value for $\phi^{c}$ by adding the superpotential

$$
\int d x_{5}\left(-\delta\left(x_{5}-L\right) \sqrt{M} J^{c} \Phi+\delta\left(x_{5}\right) \frac{\kappa}{\sqrt{M}} \Phi^{c} H_{u} H_{d}\right) .
$$

The new terms modify the equations of motion

$$
\begin{aligned}
& \Phi_{F}=-\delta\left(x_{5}-L\right) \sqrt{M} J^{c}+\left(m-\partial_{5}\right) \phi^{c}=0, \\
& \Phi_{F}^{c}=-\delta\left(x_{5}\right) \sqrt{M} J+\left(m+\partial_{5}\right) \phi=0,
\end{aligned}
$$

where we have assumed that the vacuum expectation values of $X$ and $H_{u} H_{d}$ are negligible compared to $J, J^{c} \sim M$. As mentioned in the previous Subsection this can be enforced by adding suitable brane potentials.

We see that the $\phi$ equation is unchanged, while the new source $J^{c}$ also "shines" an expectation value for $\phi^{c}$

$$
\phi^{c}=\sqrt{M} J^{c} \frac{e^{m\left(x_{5}-L\right)-m 2 \pi R \theta\left(x_{5}-L\right)}}{1-e^{-m 2 \pi R}} .
$$

Note that since we have placed the source on the supersymmetry breaking brane $\phi^{c}$ is "shined" in the opposite direction from $\phi$, as depicted in Fig. 'F- The generated $\mu$ term is equal to

$$
\mu=\frac{\kappa}{\sqrt{M}} \phi^{c}(0)=\kappa J^{c} \frac{e^{-m L}}{1-e^{-m 2 \pi R}} \sim \kappa J^{c} e^{-m L}
$$

Comparing this to the gaugino mass Eq. ( $1 / 100$.

Note that $\mu$ has the exact same exponential suppression factor $e^{-m L}$ as $m_{1 / 2}$. This follows from the fact that $\phi$ and $\phi^{c}$ reside in the same five dimensional supersymmetry multiplet. In other words, five dimensional supersymmetry relates the exponential suppression factors appearing in $\mu$ and $M_{1 / 2}$. It is disappointing that because of the volume suppression in the gaugino masses we still need to choose a small coupling $\kappa$ to get $\mu \sim M_{1 / 2}$. However, $\kappa$ is a superpotential coupling and as such can be small naturally. Note that the spatial separation of the supersymmetry breaking $X_{F}$ from the location of the Higgs fields does not allow a $B \mu$ term at the high scale. We therefore do not have the usual problem $B \sim 16 \pi \mu$ which haunts most other approaches to the $\mu$ problem. Finally, we emphasize that this new extra-dimensional solution to the $\mu$ problem does have broader applicability.

\section{Radius stabilization}

In the discussion above we have assumed that the radius $R$ of the extra dimension and the distance $L$ between the branes are fixed. In a complete theory both parameters correspond 
to fields. We now discuss a simple supersymmetry-preserving mechanism to stabilize both $R$ and $L$. Our mechanism is a trivial modification of [is]. In its simplest form it requires a single additional massive bulk hypermultiplet $\left(\Psi, \Psi^{c}\right)$ with couplings to brane fields

$$
\int d x_{5}\left(-\delta\left(x_{5}\right) \sqrt{M}\left[I \Psi^{c}+I^{c} \Psi\right]+\delta\left(x_{5}-L\right) \sqrt{M}\left[A(\Psi-\Lambda \sqrt{M})+A^{c}\left(\Psi^{c}-\Lambda^{c} \sqrt{M}\right)\right]\right) .
$$

Assuming that the brane fields $A, A^{c}$ have no vacuum expectation valuesin' one finds the following equations of motion

$$
\begin{aligned}
& \Psi_{F}=-\delta\left(x_{5}\right) \sqrt{M} I^{c}+\left(m_{\Psi}-\partial_{5}\right) \psi^{c}=0 \\
& \Psi_{F}^{c}=-\delta\left(x_{5}\right) \sqrt{M} I+\left(m_{\Psi}+\partial_{5}\right) \psi=0 \\
& A_{F}=\psi(L)-\Lambda \sqrt{M}=0, \quad A_{F}^{c}=\psi^{c}(L)-\Lambda^{c} \sqrt{M}=0
\end{aligned}
$$

which have unique supersymmetry preserving solutions for $R$ and $L$. For example for symmetric values of the parameters $\Lambda=\Lambda^{c}$ and $I=I^{c}$ we find

$$
L=\pi R=\frac{1}{m_{\Psi}} \operatorname{arcsinh}\left(\frac{I}{2 \Lambda}\right)
$$

Thus, for $I$ and $\Lambda$ of order $M$ a radius of the desired size is generated by choosing a relatively small mass for the bulk scalar $m_{\Psi} \sim M / 30$.

\section{DISCUSSION}

Minimal Gaugino Mediation is a very compelling and predictive theoretical framework which solves all supersymmetric naturalness problems without fine-tuning.

Mg̃M solves the supersymmetric flavor problem: At the high scale $M_{c}$ the scalar masses and A-terms vanish, and therefore the only flavor violation in renormalizable couplings resides in the Yukawa couplings. Gaugino loops generate universal positive scalar masses at low energies. Small non-universalities in the masses arise from the Yukawa interactions, but these contributions do not lead to new flavor violation because they are aligned with the Yukawa matrices. An exception to this is the running of the scalar masses above the GUT scale where flavor is broken by unified interactions [i] are light in Mg̃ M event rates for lepton flavor violating processes such as $\mu \rightarrow e \gamma$ might be near the experimental bounds.

\footnotetext{
${ }^{5}$ In the absence of supersymmetry breaking these expectation values are flat directions. It is straightforward to enforce the vanishing expectation values, for example by adding a brane superpotential $\delta\left(x_{5}-L\right)\left[A B+B^{2} C\right]$ for $A$ and similarly for $A^{c}$.
} 
Mg̃ M solves the supersymmetric CP problem: This is easy to understand by realizing that at the compactification scale (where $m^{2}=A=B=0$ ) the phases in $M_{1 / 2}$ and $\mu$ can be removed by phase redefinitions of the gaugino fields and the Higgs superfields, respectively. Therefore, the theory has no new phases beyond the phases in the Yukawa couplings and no supersymmetric CP violation. This does not solve the strong CP problem however.

$\mathrm{Mg} \mathrm{M}$ is very predictive and therefore testable: The model has only two free parameters which implies that there are many relations between the masses of the superpartners and Higgses which can be tested experimentally.

Mğ M has a great cold dark matter candidate: The LSP of Mg̃ M is almost a pure Bino for most of the parameter space. This makes the calculation of the relic neutralino (Bino) density relatively easy, because in this scenario neutralino annihilations are dominated by the t-channel exchange of the right-handed sleptons. If one ignores the small (but interesting) region of parameter space where the stau and neutralino are degenerate to within $5 \%$ (and

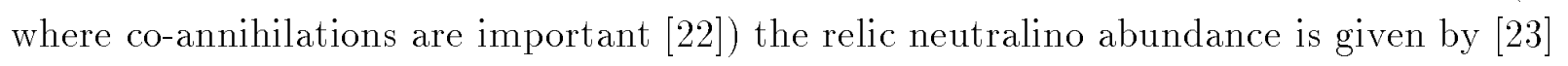

$$
\Omega_{\chi} h^{2} \approx \frac{\left(m_{\tilde{l}_{R}}^{2}+m_{\chi}^{2}\right)^{4}}{(1.4 T e V)^{2} m_{\chi}^{2}\left(m_{\tilde{l}_{R}}^{4}+m_{\chi}^{4}\right)} \approx \frac{m_{\tilde{l}_{R}}^{2}}{(480 G e V)^{2}} .
$$

This formula is accurate to about $20 \%$ over the whole parameter space plotted in Figure except for where neutralinos and staus are almost degenerate (a narrow band surrounding

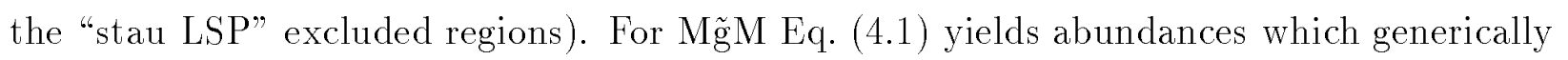
are cosmologically safe and often lie within the cosmologically interesting regime $0.1<$ $\Omega_{\chi} h^{2}<0.3$ as is evident from Figure $\sum_{-i}$

Mg̃ M is theoretically well motivated: Separation of SUSY breaking and the MSSM matter fields onto two different branes naturally gives rise to the Gaugino Mediation boundary condition. If the Higgs fields also live on the MSSM matter brane then all supersymmetry breaking soft Higgs mass parameters vanish, giving Mg̃ M. The model is very economical and unifies. We believe that the model is sufficiently "conservative", successful in solving all the problems of supersymmetry, and elegant that it has a real chance of describing Nature.

\section{ACKNOWLEDGMENTS}

We thank Nima Arkani-Hamed, Howard Baer, Howie Haber, Markus Luty, Graham Kribs, Kirill Melnikov, Michael Peskin, and Jim Wells for useful discussions. MS is supported

by the DOE under contract DE-AC03-76SF00515. WS is supported by the DOE under contract DOE-FG03-97ER40506. 


\section{REFERENCES}

[1] L. Randall and R. Sundrum, hepeth79810155:

[2] G.F. Giudice, M.A. Luty, H. Murayama, and R. Rattazzi, JHEP 9812:027 (1998), thep-ph $[9810442$.

[3] A. Pomarol and R. Rattazzi, JHEP 9905: 013 (1999), hep-ph [9903448;

Z. Chacko, M. Luty, I. Maksymyk, and E. Pontón, hep-ph 29905390 's

E. Katz, Y. Shadmi, and Y. Shirman, JHEP 9908: 015 (1999), hep-ph $\overline{2} \overline{9} \overline{0} \overline{6} \overline{2} \overline{9} \overline{6}_{*}^{\prime}$

[4] D.E. Kaplan, G.D. Kribs, and M. Schmaltz, hep-ph [9911 $29 \overline{9}$

[5] Z. Chacko, M. Luty, A.E. Nelson, and E. Pontón, JHEP 0001: 003 (2000), thep-ph $[9911323$

[6] E.A. Mirabelli and M.E. Peskin, Phys. Rev. D58 (1998) 065002, hepth/9712214

[7] J. Ellis, C. Kounnas, and D.V. Nanopoulos, Nucl. Phys. B247 (1984) 373;

A.B. Lahanas and D.V. Nanopoulos, Phys. Rep. 145 (1987) 1.

[8] N. Arkani-Hamed, L. Hall, D. Smith, and N. Weiner, he-p-ph [99114211.

[9] D. Castano, E. Piard, and P. Ramond, Phys. Rev. D49 (1994) 4882, hepeph

[10] V. Barger, M.S. Berger, and P. Ohmann, Phys. Rev. D49 (1994) 4908, he-p-ph [931126\%.

[11] H. Baer, J. Gunion, C. Kao, and H. Pois, Phys. Rev. D51 (1995) 2159, ihep-ph [9406374.

[12] M. Schmaltz and W. Skiba, to appear.

[13] R. Barbieri and L.J. Hall, Phys. Lett. B338 (1994) 212, hepeph

R. Barbieri, L.J. Hall, and A. Strumia, Nucl. Phys. B445 (1995) 219, thep-ph [9501334.

[14] S.P. Martin and M.T. Vaughn, Phys. Rev. D50 (1994) 2282, hep-ph [9311340'

[15] H.E. Haber and R. Hempfling, Phys. Rev. D48 (1993) 4280, hep-ph

[16] D. Pierce, J. Bagger, K. Matchev, and R. Zhang, Nucl. Phys. B491 (1997) 3, thep-ph 69606211 .

[17] A. Blondel, talk given at LEPC meeting representing the Aleph collaboration, Nov. 1999,

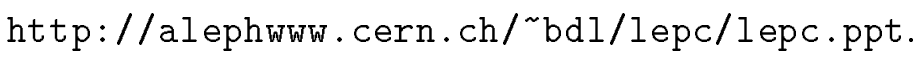

[18] Higgs working group of the Physics at Run II, http://fnth37.fnal.gov/higgs.html.

[19] Report of SUGRA Working Group for Run II of the Tevatron, http://fnth37.fnal.gov/sugra/sugra.ps. 
[20] F. Gabbiani, E Gabrielli, A. Masiero, and L. Silvestrini, Nucl. Phys. B477 (1996) 321, thep-ph 960438

[21] J. Hisano, T. Moroi, K. Tobe, and M. Yamaguchi, Phys. Lett. B391 (1997) 341, er-

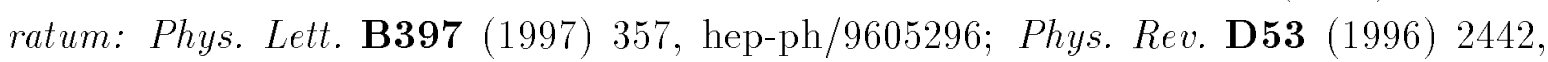
thep-ph $[9510309$.

[22] J. Ellis, T. Falk and K. A. Olive, Phys. Lett. B444 (1998) 367, hiep-ph

[23] J.D. Wells, Phys. Lett. B443 (1998) 196, "hep-ph[9809504;

M. Dress and M. Nojiri, Phys. Rev. D47 (1993) 376, thep-ph $29207 \overline{2} \overline{3}$ 